\title{
An Evaluation of the Possible Protective Effects of Neonatal Striatal Transplants Against Kainic Acid-Induced Lesions
}

\author{
David R. Nash, Stanley M. Kaplan, \\ Andrew B. Norman and Paul R. Sanberg \\ Division of Neuroscience, Departments of Psychiatry, Neurosurgery, \\ Psychology, Anatomy and Physiology \\ University of Cincinnati College of Medicine \\ Cincinnati, Ohio 45267-0559, U.S.A. \\ and Cellular Transplants, Inc., 4 Richmond Square, Providence, RI 02906, U.S.A.
}

\section{ABSTRACT}

The present study examined the recent report that transplantation of neonatal striatal tissue into kainic acid (KA) lesioned striatum protected the contralateral striatum from a subsequent KA lesion. We did not find a significant difference in the survival rate of animals that received neonatal striatal transplants into a KA lesioned striatum followed by a subsequent lesion of the contralateral striatum compared to those animals that received bilateral KA-induced striatal lesions alone. The tissue transplants did not protect against the degeneration of striatal neurons induced by KA. Indeed, the survival rate was very low (25\%) in the transplant groups. A second experiment was also performed to examine whether a neonatal striatal transplant might reduce the severe syndrome of aphagia and adipsia associated with KA lesions of the striatum. Animals that received the neonatal striatal transplants showed increased aphagia and adipsia compared to animals only receiving the KA lesion. Again, the transplant group had a very low survival rate (10\%). The present study was unable to confirm that neonatal striatal transplants protect against KA lesions either by themselves or in conjunction with a recent $\mathrm{KA}$ lesion.

Reprint address:

Paul R. Sanberg, Ph.D.

Cellular Transplants, Inc.

4 Richmond Square

Providence, RI 02906, U.S.A.
Key words: Striatum, Huntington's disease, kainic acid, neural transplants.

Neural tissue transplants represent a potential approach to treating brain injury and neurodegenerative disorders in humans. They have been shown to reverse the behavioral deficits observed in several animal models of neurodegenerative diseases $14,8,10 /$. Earlier research has utilized kainic acid (KA) injections into the striatum of adult rats to create a model with many of the anatomical and behavioral abnormalities seen in patients with Huntington's disease (HD) $/ 13,14 /$. In adult rats, $\mathrm{KA}$ is an excitotoxin which selectively destroys neuronal perikarya, while leaving axons and terminals intact $/ 13 \%$. It has been postulated that an endogenous excitotoxin with some properties similar to KA may be responsible for the neurodegeneration associated with $\mathrm{HD} / 1,15 /$. In order for neural transplantation to become clinically useful, it must be determined whether the transplanted tissue will be affected by the underlying neurodegenerative process.

Recently, Tullipan et al. /16,17/ suggested that neonatal striatal transplants would protect against lesions by the excitotoxins, KA and quinolinic acid. Neonatal grafts were used because of their apparent resistance to KA toxicity both in vivo and in vitro $/ 3,7,18,19 /$. They postulated that there was a neurohumoral influence provided either by the grafted tissue, the lesioned striatum, or by an interaction between the graft and the lesioned host tissue which served to protect against excitotoxic lesions. Because of the potential clinical importance, the present study attempted to 
replicate this finding, and test whether the transplant functions alone or in conjunction with the lesioned striatum.

Two separate experimental protocols were utilized in these studies. The design of the first experiment was based upon the work of Tullipan et al. /16/. Male Sprague-Dawley rats weighing 240-300 g were divided into 4 groups: 1) bilateral striatal KA lesioned, each lesion separated by 3 days $(\mathrm{n}=8)$; 2) KA lesion of one striatum followed 3 days later by neonatal striatal transplant of the ipsilateral striatum and KA lesion of the contralateral striatum $(n=8) ; 3) \mathrm{KA}$ lesion of one striatum followed 3 days later by neonatal cortex transplant into the ipsilateral striatum and KA lesion of the contralateral striatum $(n=8) ; 4)$ sham lesion followed 3 days later by sham transplant of the ipsilateral striatum and sham lesion of the contralateral striatum $(n=8)$. All rats were anesthetized with chloral hydrate $(400 \mathrm{mg} / \mathrm{kg}$, i.p.) and then stereotaxically positioned. All KA lesions used 10 nmol KA in 2 ul of phosphate buffered saline (PBS) $(\mathrm{pH}=7.4)$. The $\mathrm{KA}$ was injected over a two minute period and the needle was left in place an additional 2 minutes. The KA was injected into the striatum of the adult rat using the following coordinates: $1.5 \mathrm{~mm} \mathrm{AP}, 2.6 \mathrm{~mm}$ $\mathrm{ML}$ relative to bregma and $4.8 \mathrm{~mm} \mathrm{DV}$ from dura /11/. All transplanted striatal tissue was obtained from 1-4 day neonatal rat pups. The rat pups were decapitated, the skin and calvarium removed and the whole striatum dissected. Whole sections of neonatal striatum were aspirated into a glass capillary tube affixed to a hamilton syringe. The solid neonatal graft (2-4 ul) was then implanted into the striatum of the adult rat using the same coordinates as above $/ 2 /$. The tissue was injected over two minutes with the needle remaining in place for an additional 2 minutes. The tissue preparation and transplant operation was performed under sterile conditions. The time from dissection until transplantation was less than 15 minutes in all animals. All animals received $4 \mathrm{ml}$ of $5 \%$ dextrose on the day of surgery and for the following 3 days, in addition to standard rat chow and water. Animals were weighed daily for two weeks following the surgery.

Gross histological examination was performed on the surviving animals from the above experiment. The rats were anesthetized and then perfused with normal saline for 5 minutes followed by a $10 \%$ formalin solution. The brains were removed and stored in $20 \%$ sucrose in $0.9 \mathrm{M}$ phosphate buffer. The fixed brains were prepared for sectioning by freezing with $\mathrm{CO}_{2}$ and a Leitz microtome was used to obtain 20 micron coronal sections. The slides were prepared for staining with cresyl violet $/ 11 /$.

Animals from the first study which attempted to replicate the work of Tullipan et al. $/ 16 /$ had an extremely high mortality rate. By one week, postoperative, only 1 of 8 animals (group 1) receiving the bilateral $\mathrm{KA}$ lesion and no transplant had survived. Similarly, groups of 8 animals receiving either the striatal (group 2) or cortex (group 3) transplants had only 2 of 8 survivors per group at 1 week post-operatively. The control group had 7 of 8 animals survive. There was no significant difference in mortality rate between the transplanted animals and the bilaterally lesioned animals. As shown in Table 1, the control animals that received sham lesions and a sham transplant had a significantly lower mortality rate than either the transplanted animals $(\mathrm{z}=2.52, \mathrm{p}<0.005)$ or the bilaterally lesioned animals $(z=3.01, p<0.001$; test for significance of proportions).

There was a bilateral shrinkage of the striatal parenchyma and an enlargement of the lateral ventricles apparent in both the animals that received bilateral $\mathrm{KA}$ lesions and the animals that received the KA striatal lesion followed by an ipsilateral neonatal striatal transplant and a contralateral striatal KA lesion (see Figure 1). The extent of the KA-induced lesions of the striatum were similar regardless of whether the animal received a neonatal striatal transplant. Also, consistent with the findings of previous studies $/ 16 /$, there was little evidence for survival of the neonatal graft. In some cases, however, the graft could be seen as depicted in Fig. 1.

In the second experiment a variation of Tullipan et al.'s /16/ experimental protocol was used. Male Sprague-Dawley rats weighing 210$240 \mathrm{~g}$ were divided into an experimental group $(n=10)$ and a control group $(n=9)$. Animals were anesthetized and positioned in the stereotaxic frame as in the previous experiment. In the experimental group, animals received a neonatal striatal transplant into an unlesioned striatum using the same coordinates and procedures as in the previous experiment. 
TABLE 1. The effect of neonatal striatal transplants on the mortality of animals receiving kainic acid lesions*

\section{Experiment I}

\begin{tabular}{lc}
\multicolumn{1}{c}{ Group } & Survival Rate \\
\hline Bilateral Lesion & $1 / 8$ \\
Lesion/Striatal Trans.-Lesion & $2 / 8$ \\
Lesion/Cortical Trans.-Lesion & $2 / 8$ \\
Sham Lesion/Sham Trans.- & \\
$\quad$ Sham Lesion & $7 / 8$
\end{tabular}

Experiment II

$\begin{array}{ll}\text { Striatal Trans. \& Lesion } & 1 / 10 \\ \text { Sham Trans. \& Lesion } & 5 / 9\end{array}$

* In experiment I animals were divided into 4 groups: 1) bilateral striatal lesion, each lesion separated by 3 days; 2) $\mathrm{KA}$ lesion of one striatum followed 3 days later by ipsilateral neonatal striatal transplant and KA lesion of the contralateral striatum; 3) same as group 2 except cortex transplants were substituted for striatal, and 4) sham lesion of one striatum followed 3 days later by an ipsilateral sham transplant and sham lesion of the contralateral striatum. Animals that received transplants had no significant difference in mortality from those animals that received bilateral striatal KA lesions. Animals in the sham group that received sham lesions and a sham transplant had a significantly lower mortality rate than either the bilateral lesion group $(\mathrm{z}=3.01, \mathrm{p}<0.001)$ or the transplant groups $(\mathrm{z}=2.56, \mathrm{p}<0.005$; test for significance of proportions). In Experiment II rats in the experimental group received a transplant of neonatal striatal tissue into one striatum and simultaneously received a 10 nmole $\mathrm{KA}$ lesion in the contralateral striatum. Animals in the control group received a sham transplant with lactated Ringers solution and a KA lesion of the contralateral striatum. Animals that received striatal transplants had a significantly increased mortality compared to the control group $(\mathrm{z}=2.12, \mathrm{p}<0.02$; test for significance of difference between two proportions).

Immediately following the transplant these animals received an injection of KA $(10 \mathrm{nmol})$ into the contralateral striatum. The control group received a sham transplant with lactated ringers $(2-4 \mathrm{ul})$ in place of the neonatal tissue. Immediately following the sham transplant these animals received an injection of $\mathrm{KA}(10 \mathrm{nmol})$ into the contralateral striatum. All animals in this experiment received the same post-operative treatment as in the first experiment.

In the second experiment all animals in both groups demonstrated both barrel rotations and seizure activity in the immediate post-operative
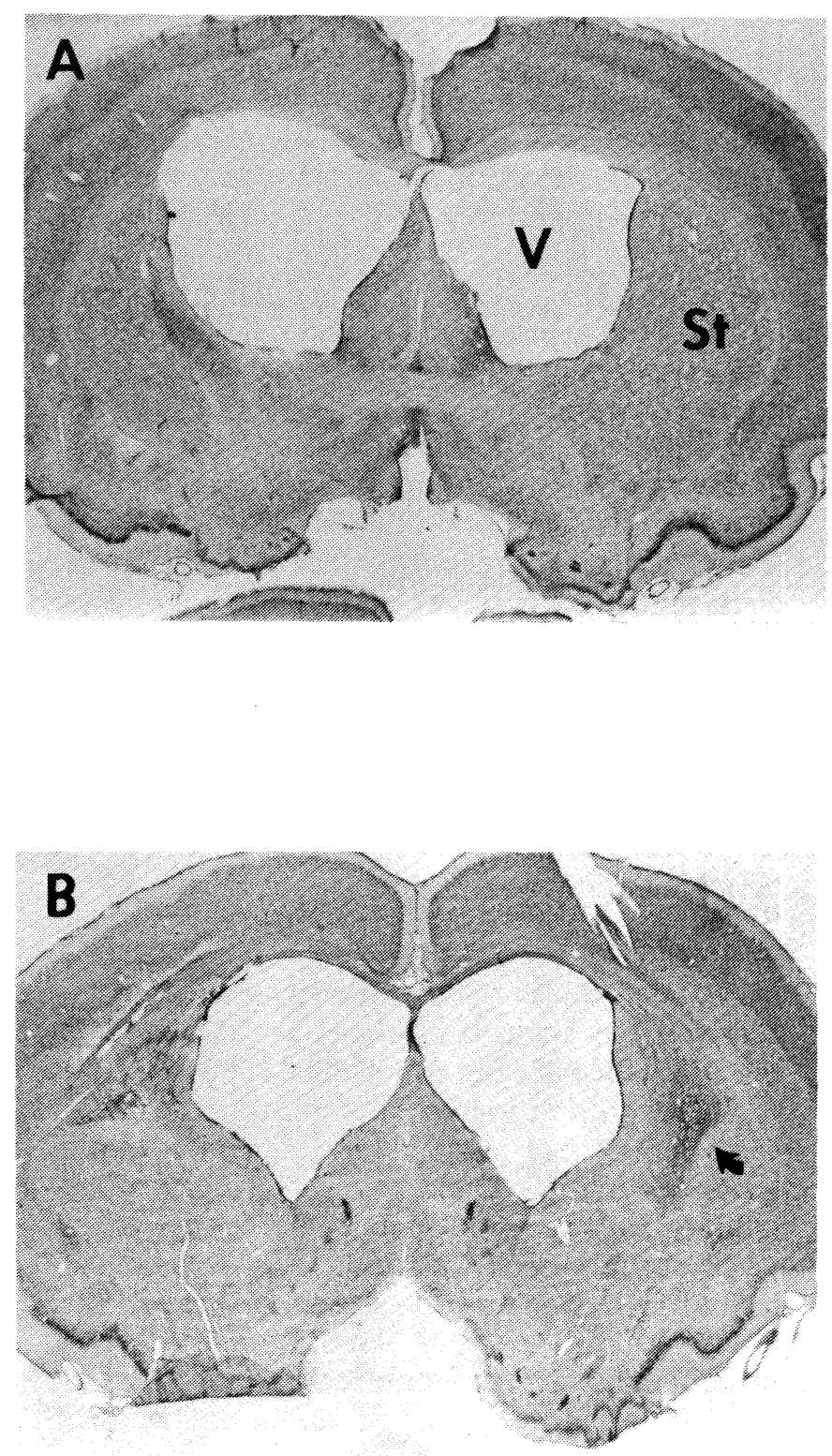

Fig. 1: (A) Photomicrograph of coronal section of a representative rat brain which received bilateral 10nmole KA lesions of the striatum (St) 3 days apart. (B) Photomicrograph shows an animal that received a $\mathrm{KA}$ lesion of the left striatum followed 3 days later by a neonatal striatal transplant and lesion of the contralateral striatum. Note the similar extent of the KA-induced damage in the two brains, as evidenced grossly by the dilation of the ventricles $(V)$ and shrinkage of the striatum (St). Arrow points to the neonatal transplant. 
period suggesting a successful unilateral lesion. In the experimental group (neonatal striatal transplant into one striatum followed immediately by a KA lesion of the contralateral striatum) 6 animals died within the first week of the surgery apparently due to the syndrome of aphagia and adipsia. The average pre-operative weight was $216 \mathrm{~g}$ in the experimental group and by day 4 the average weight had fallen to $176 \mathrm{~g}$. This represented a $19 \%$ fall in body weight. By the end of the second week nine out of ten of the rats which had received striatal transplants had died. In the control group (sham transplant into one striatum followed immediately by a KA lesion of the contralateral striatum) 3 animals died within the first week of surgery. In this group, however, the syndrome of aphagia and adipsia was much less severe. Animals had an average pre-op weight of $234 \mathrm{~g}$. The rats reached a nadir for weight on day 3 down to $207 \mathrm{~g}$ (Figure 2). This represented only a $12 \%$ loss in weight. By the end of the second week only 4 out of 9 of control animals that had received a sham transplant had died. Therefore, animals that received the striatal transplant had a significantly higher mortality compared to animals that received the sham transplant $(\mathrm{z}=2.12, \mathrm{p}<0.02$ test of significance of proportions) see Figure 2.

In the present study we demonstrated that the transplants either alone or in conjunction with a lesioned striatum failed to protect against the lethal effects of $\mathrm{KA}$ injected into the striatum, the syndrome of aphagia and adipsia, and the histological changes also associated with $\mathrm{KA}$ lesions of the striatum. We found that the experimental protocol reported by Tullipan et al /16/ produced death in virtually all animals regardless of whether they received a neonatal striatal transplant. Possibly the extremely toxic effect of the large dose of KA masked whatever protective effect may have been present. Additionally, the mortality rate was further increased by the stress of two surgical procedures, thereby confounding the probability of demonstrating any protective effect. Although our findings are convincing, a number of notable differences exist between our study and that of Tullipan et al /16/. First a difference in rat sex, strain and weight may explain the increase in mortality from KA lesions in our experiments /12/. While we used male Sprague-Dawley rats weighing between 240-300 g, Tullipan's group used female Lewis rats weighing between 175-

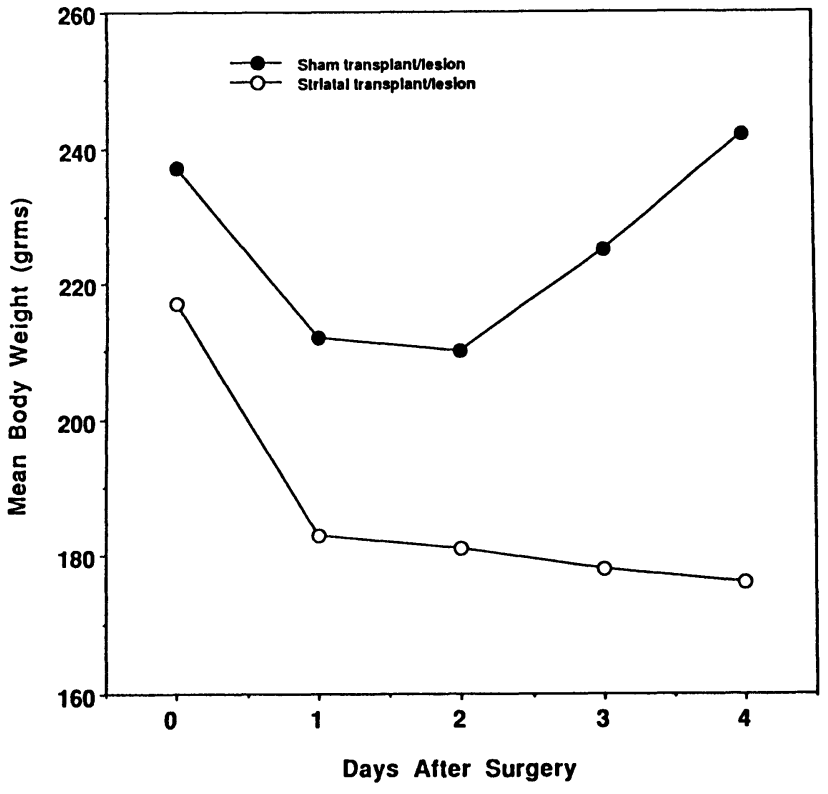

Fig. 2: Effect of transplants on KA-induced body weight loss. Animals from Experiment II were weighed daily to determine if the transplant protects from the KA-induced syndrome of aphagia and adipsia. Animals that recelved transplants actually lost more weight and took longer to recover than animals that received sham transplants instead of striatal transplants.

$200 \mathrm{~g}$. KA dose and donor age were comparable. A second factor could be a difference in the neurotoxicity of two separate batches of KA. Furthermore, in attempting to duplicate this work our rats received no special post-operative care other than $4 \mathrm{ml}$ of $5 \%$ dextrose for 3 days. If the animals in previous studies received some post-op care, e.g. tube feedings or food mash preparations, then this may have also influenced animal survival.

In our second experiment we showed that transplants actually increased mortality in experiments where animals received only the neonatal striatal transplants and simultaneous contralateral lesions with $\mathrm{KA}$. In this case the transplants themselves could have damaged one striatum while the KA damaged the other striatum and, thereby, contributed to the increase in mortality /6/. Furthermore, animals which received striatal transplants showed a more profound syndrome of aphagia and adipsia suggesting these animals sustained more striatal 
damage than those animals that only received sham transplants.

The histological evidence from the first experiment also served to demonstrate that the transplants failed to protect against KA lesions. The animals that received striatal transplants had no protection in terms of size of the KAinduced lesions. Interestingly, as in previous studies $/ 16 /$, there was no evidence of the solid neonatal striatal graft.

Recently, Levivier et al / $9 /$ found that fetal striatal transplants could protect against the apomorphine-induced turning behavior that results from unilateral quinolinic acid lesions of the pre-transplanted striatum. However, histological analysis of their animals did not reveal any protection in terms of the size of the lesion, similar to the present results. It is possible that the protection of the behavioral deficits seen by Levivier et al /9/ may be due to some protective factor as reported by Tullipan et al /16/. However, apomorphine-induced rotation can be affected by a number of different variables and can even be reduced by larger striatal lesions, per se $15 \%$. Therefore, further work should be done with other behavioral paradigms to clarify this issue.

The present study strongly suggests that there is no protective effect of neonatal transplants at high doses of KA. Because of the potential clinical significance of this area, it would be interesting to look for possible protective effects of transplants on the behavioral and morphological effects of lower doses of excitotoxins.

\section{REFERENCES}

1. Beal MF, Kowall NW, Ellison DW, Mazurek MF, Schwartz KJ, Martin JB. Replication of the neurochemical characteristics of Huntington's disease by quinolinic acid. nature 1986; 321: 168-171.

2. Bjorklund A, Stenevi U, Schmidt RH, Dunnet SB, Gage FH. Intracerebral grafting of neuronal cell suspensions. I. Introduction and general methods of preparation Acta Physiol Scand (Suppl) 1983 522: 17.

3. Campochiaro P, Coyle JT. Ontogenetic development of kainate neurotoxicity: correlates with glutaminergic innervation. Proc Natl Acad Sci USA 1978; 75: 2025 2029.

4. Deckel AW, Robinson RG, Coyle JT, Sanberg PR. Reversal of long term locomotor abnormalities in the kainic acid model of Huntington's disease by 18 day fetal striatal implants. Eur J Pharmacol 1983; 93: 287. 288.

5. Friehs GM, He LS, Parker RG, Ojakangas CL, Turner DA, Haines SJ, Ebner TJ. Comparison of caudate lesioning and adrenal medulla grafting in 6OHDA rats. Soc Neurosci Abs 1989; 15: 490.

6. Hagenmeyer-Houser SH, Sanberg PR. Locomotor behavior changes induced by E-17 striatal transplants in normal rats. Pharmacol Biochem \& Behav 1987; 27: 583-586.

7. Hattori T, McGeer EG. Fine structural changes in the rat striatum after local injections of kainic acid. Brain Res 1977; 129: 174-180.

8. Isacson $O$, Brundin PM, Keldy PAT, Gage FH, Bjorklund A. Functional neuronal replacement by grafted striatal neurons in the ibotenic acid lesioned rat striatum. Nature 1984; 311: 458-460.

9. Levivier $M$, Sladek JR, Collier T, HagenmeyerHouser SH, Gash DM. The protective effect of various striatal implants on intrastriatal quinolinic acid lesion. Restor Neurol Neurosci, Suppl 1989; 1: 50.

10. Norman $A B$, Lehman MN, Sanberg PR. Functional effects of fetal striatal transplants. Brain Res Bull 1989; 46: 163-171.

11. Paxinos G, Watson C. The Rat Brain in Stereotaxic Coordinates. Sydney: Academic, 1984.

12. Sanberg PR, Pisa M, McGeer EG. Strain differences and kainic acid neurotoxicity. Brain Res 1979; 166: 431-435.

13. Sanberg PR, Fibiger HC. Body weight feeding and drinking behaviors in rats with kainic-acid induced lesions of striatal neurons with a note on body weight symptomatology in Huntington's disease. Expt Neurol 1979; 66: 444-446.

14. Sanberg PR, Coyle JT. Scientific approaches to Huntington's disease. CRC Critical Review Clin Neurobiol 1984; 1: 1-44.

15: Schwarcz R, Okuno E., White RJ, Bird ED, Whetsell WO. 3-Hydroxyanthranilate oxygenase activity is increased in the brains of Huntington disease victims. Proc Natl Acad Sci 1988; 85: 4079-4090.

16. Tullipan N, Huang S, Whetsell WO, Allen GS. Neonatal striatal grafts prevent lethal syndrome produced by bilateral intrastriatal injection of kainic acid. Brain Res 1986; 377: 163-167.

17. Tullipan N, Luo S, Allen GS, Whetsell WO. Striatal grafts provide sustained protection from kainic and quinolinic acid-induced damage. Exp Neurol 1988; 102: 325-332.

18. Whetsell WO, Schwarcz R. Mechanisms of excitotoxins examined in organotypic cultures of rat central nervous system. In: Fuxe $K$, Roberts $P$, Schwarcz R (eds.), Excitotoxins, Wenner-Gren Sympoxia Series, Symposium No. 39. London: Macmillan Press, 1983.

19. Whetsell WO, Ecob-Johnston MS, Nicklas WJ. Studies of kainate-induced caudate lesions in organotypic tissue culture. Adv Neurol 1979; 23: 645654. 

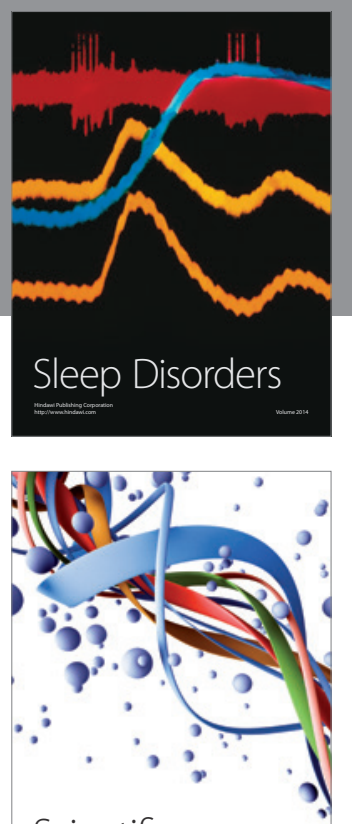

Scientifica
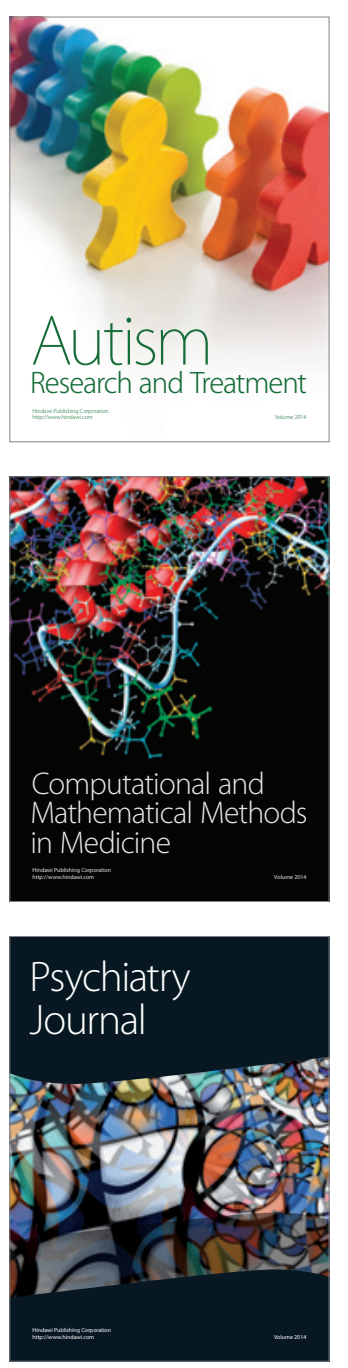
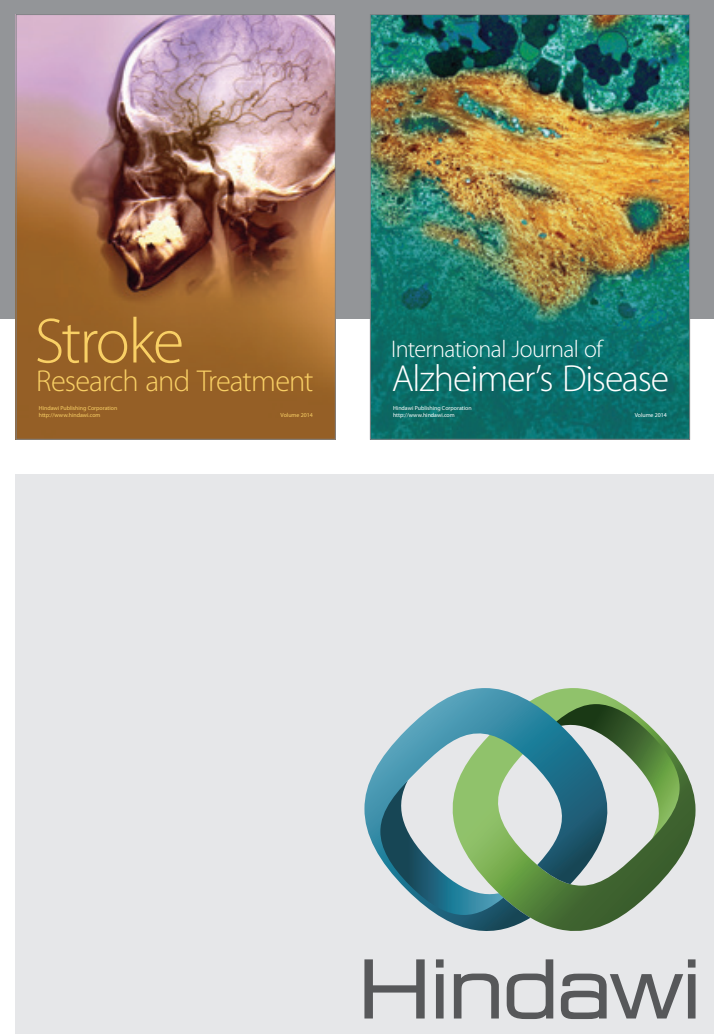

Submit your manuscripts at

http://www.hindawi.com
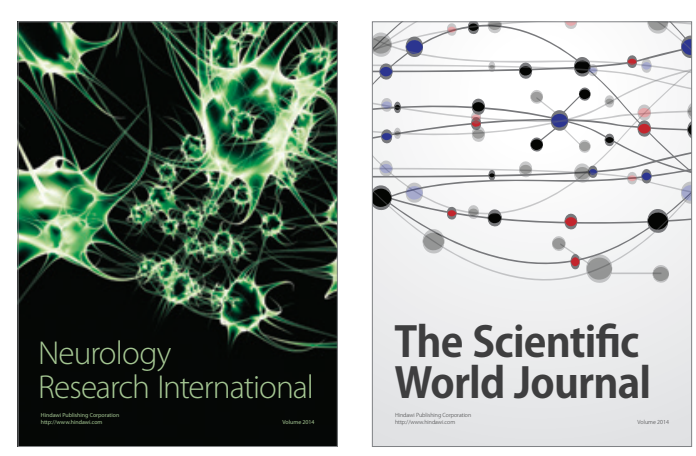

The Scientific World Journal

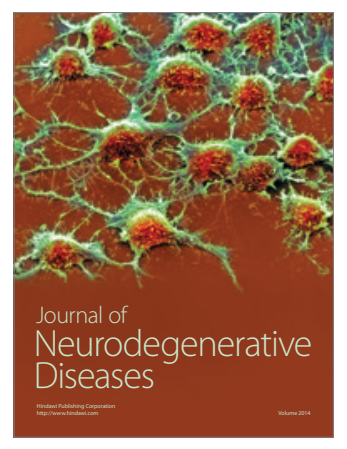

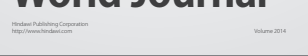

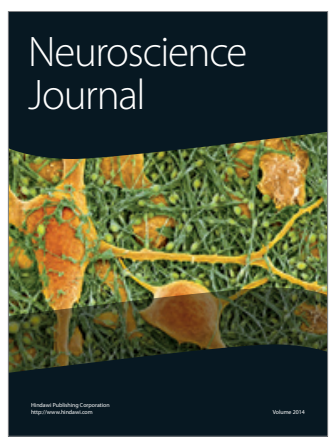

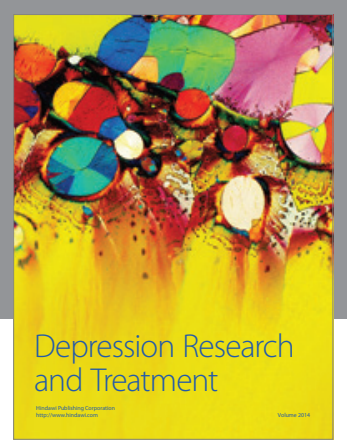
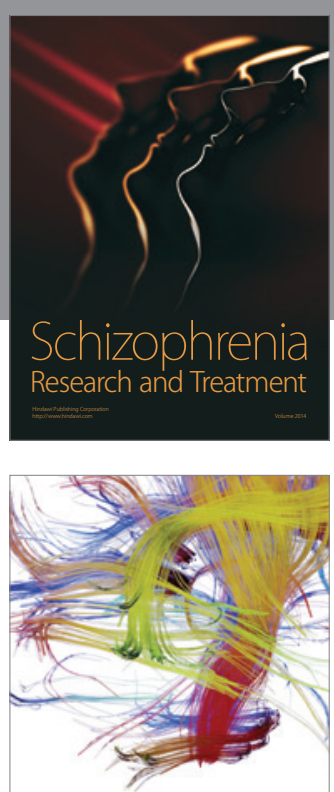

Brain Science

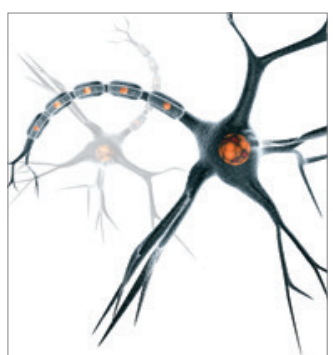

Neural Plasticity
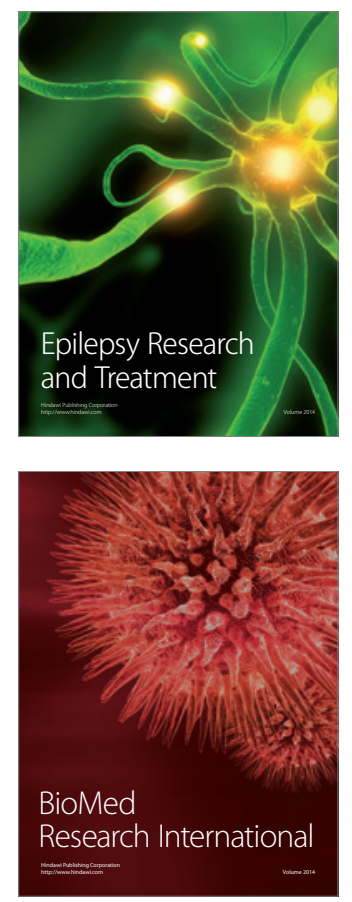

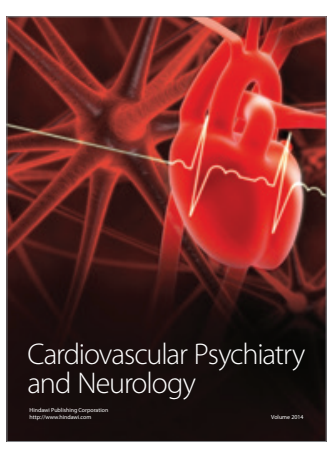

Parkinson's

Disease
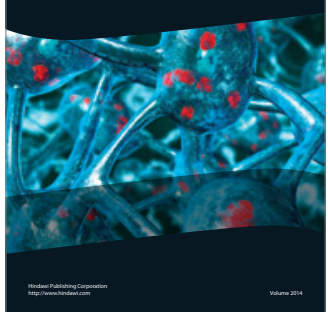